\title{
Distributions of positive mass, which maximize a certain generalized energy integral
}

\author{
By Göran Björck
}

Introduction. The classical theory of the equilibrium distribution for the Newtonian kernel deals with the problem of finding and studying distributions of positive mass with a given total mass, on a compact set $F$ in $n$-dimensional Euclidean space $(n>2)$, which minimize the energy integral $\int_{F}|x-y|^{2-n} d \mu(x) d \mu(y)$ where $|x-y|$ is the Euclidean distance between the points $x$ and $y$. M. RIEsz and Frostman have generalized this to the case of the " $\alpha$-potentials" with the energy integral $\int_{F} \int_{F}|x-y|^{\alpha-n} d \mu(x) d \mu(y),(0<\alpha \leq 2)$. If we want to study a similar problem for the "energy integral" $\int_{F} \int_{F}|x-y|^{\lambda} d \mu(x) d \mu(y)$ with $\lambda>0$, we must evidently turn our interest to distributions that maximize this integral. Let us call such distributions maximal distributions. The purpose of this paper is to investigate for which values of $\lambda>0$ there always exists a unique maximal distribution and to study the properties of maximal distributions.

The value of the "energy integral" with arbitrary $\lambda$ has been studied by Pósya and SzEgö [3]. They studied, in particular, the special case when $F$ is a sphere or a solid sphere.

Frostman [1, p. 39] has proved that, in the case of the $\alpha$-potentials, that distribution which minimizes the energy integral has certain other extremum properties. For maximal distributions the corresponding properties do not hold, as is shown in theorems 13-15.

Our main results are the following: For any $\lambda>0$, the potential of a maximal distribution is constant in the support of the distribution and less than or equal to this constant value in $F$. For any $\lambda>0$, the mass of a maximal distribution lies on the boundary of $F$. For $\lambda>1$, it is in the extreme points of the convex hull of $F$. For $\lambda<2$, there is a unique maximal distribution. For $\lambda>2$, any maximal distribution consists entirely of point-masses, not more than $(n+1)$ in number.

Notation and definitions. Let $F$ be a compact set in the $n$-dimensional Euclidean space $R_{n}$ with $n \geq 1$. Denote the points of $R_{n}$ by $x, y$, etc. Let subscripts indicate different points and superseripts the coordinates of any one point, e.g. $y_{i}=\left(y_{i}^{1}, y_{i}^{2}, \ldots, y_{i}^{n}\right)$. Denote by $|x-y|$ the Euclidean distance, so that 


\section{G. BJöRCK, Distributions of positive mass}

$|x-y|^{2}=\sum_{k=1}^{n}\left(x^{k}-y^{k}\right)^{2}$. Let $m=m_{F}$ be the class of all non-negative mass distributions on $F$ with total mass equal to unity, that is, the class of all completely additive set-functions $\mu$ which take non-negative values on all Borel subsets of $R_{n}$ and which satisfy $\mu(F)=1$ and $\mu\left(R_{n}-F\right)=0$. Let $\lambda$ be a positive number. For any pair $\left(\mu_{1}, \mu_{2}\right)$ of mass distributions on $F$, and for any mass distribution $\mu$ on $F$, define, as follows, the functionals $I\left(\mu_{1}, \mu_{2}\right)$ and $I(\mu)$, respectively:

$$
I\left(\mu_{1}, \mu_{2}\right)=\iint_{F}|x-y|^{\lambda} d \mu_{1}(x) d \mu_{2}(y), \quad I(\mu)=I(\mu, \mu) .
$$

The potential $p_{\mu}(x)$ of a distribution $\mu$ is defined as

$$
p_{\mu}(x)=\int_{F}|x-y|^{2} d \mu(y)
$$

Evidently,

$$
I\left(\mu_{1}, \mu_{2}\right)=\int_{F} p_{\mu_{1}}(x) d \mu_{2}(x)=\int_{F} p_{\mu_{2}}(x) d \mu_{1}(x) .
$$

The non-negative number $M=M_{F}$ is defined as

$$
M=\sup _{\mu \in \mathfrak{m}} I(\mu) .
$$

A maximal distribution is defined as a distribution $\mu$, such that

$$
\mu \in M \text { and } I(\mu)=M \text {. }
$$

The distribution consisting of the unit mass in the point $x$ is denoted by $\delta_{x}$.

Theorem 1. For every $F$ and every $\lambda>0$ there exists a maximal distribution.

Proof: Let $\left\{\mu_{n}\right\}$ be a sequence such that $\mu_{n} \in \mathcal{M}$ and $I\left(\mu_{n}\right) \rightarrow M$. Select from this sequence a sub-sequence $\left\{\mu_{n_{i}}\right\}$, converging weakly to a distribution $\mu^{*}$ (i.e. $\int_{F} f(y) d \mu_{n_{i}}(y) \rightarrow \int_{F} f(y) d \mu^{*}(y)$ for every continuous $f$ ). Since $|x-y|^{2}$ is continuous in $x$ and in $y$, we get $I\left(\mu_{n_{i}}\right) \rightarrow I\left(\mu^{*}\right)$ (see e.g. Frostman [1, p. 17]). Hence $I\left(\mu^{*}\right)=M$. But $\mu^{*}$, being a weak limit of elements of $m$, must belong to $m$. Hence $\mu^{*}$ is maximal, which completes the proof.

Theorem 2. If $\mu$ is a maximal distribution with support $F_{1}$, then $p_{\mu}(y)=M$ for $y \in F_{1}$, and $p_{\mu}(y) \leq M$ for $y \in F$.

Proof: Let $v$ be a mass distribution on $F$, satisfying:

$$
\left.\begin{array}{c}
\qquad(F)=0 \\
\mu+\varepsilon \cdot \nu \geq 0 \text { for every } \varepsilon \text { in the interval } 0 \leq \varepsilon \leq 1 \\
(\mu \text { is the given maximal distribution) }
\end{array}\right\} .
$$


It follows that $(\mu+\varepsilon \cdot \nu) \in M$, and hence

$$
M \geq I(\mu+\varepsilon \cdot v)=I(\mu)+\varepsilon^{2} I(v)+2 \varepsilon I(\mu, v)=M+\varepsilon^{2} I(v)+2 \varepsilon I(\mu, v) .
$$

Thus, for every $\varepsilon$ with $0 \leq \varepsilon \leq 1$,

which implies

$$
0 \geq \varepsilon \cdot(2 I(\mu, v)+\varepsilon \cdot I(v)),
$$

$$
I(\mu, v) \leq 0 .
$$

Suppose now that the theorem does not hold, even if $M$ is replaced by some other constant $C$. There is then a point $y_{1}^{\prime} \in F_{1}$ and a point $y_{2} \in F$, such that

$$
a=p_{\mu}\left(y_{2}\right)>p_{\mu}\left(y_{1}\right)=b \text {. }
$$

Let $K$ be a solid sphere ${ }^{1}$ with centre $y_{1}$ and so small that $y_{2} \notin K$ and the oscillation of $p_{\mu}$ in $K$ is less than or equal to $\frac{a-b}{2}$. Since $y_{1} \in F_{1}, m=\mu(K)>0$. Define, as follows, the distribution $v$ which "moves the mass from $K$ to $y_{2}$ ":

$$
\nu(e)=m \cdot \delta_{y_{1}}(e)-\mu(e \cap K) .
$$

Evidently $y$ satisfies (1), and hence (2) is true. But

$$
\begin{aligned}
I(\mu, v)=\int_{F} p_{\mu}(x) d v(x)=p_{\mu}\left(y_{2}\right) \cdot & m-\int_{K} p_{\mu}(x) d \mu(x) \\
& \geq a \cdot m-\left(b+\frac{a-b}{2}\right) \cdot m=\frac{a-b}{2} \cdot m>0,
\end{aligned}
$$

which is contrary to (2). Hence $p_{\mu}(y)$ is constant $=C$ for $y \in F_{1}$, and $p_{\mu}(y) \leq C$ for $y \in F$. Consequently,

$$
M=I(\mu)=\int_{F} p_{\mu}(x) d \mu(x)=\int_{F_{1}} p_{\mu}(x) d \mu(x)=C \int_{F_{1}} d \mu(x)=C,
$$

which completes the proof.

Corollary. If $\lambda=2, F_{1}$ is a subset ${ }^{2}$ of a sphere.

We have

$$
p_{\mu}(y)=\int_{F_{1}} \sum_{i=1}^{n}\left(x^{i}-y^{i}\right)^{2} d \mu(x)=\sum_{i=1}^{n}\left(y^{i}\right)^{2}+\int_{F_{1}} \sum_{i=1}^{n}\left(x^{i}\right)^{2} d \mu(x)-2 \sum_{i=1}^{n} y^{i} \int_{F_{1}} x^{i} d \mu(x)
$$

and if $y \in F_{1}$, we get $p_{\mu}(y)=M$, which is the equation of a sphere.

\footnotetext{
1 We call the set of points $x$ which satisfy $\left|x-y_{1}\right|=R$ a sphere, and the set of points which satisfy $\left|x-y_{1}\right| \leq R$ a solid sphere.

2 The inclusion sign and the concept of subset are used in the broad sense (equality not excluded).
} 


\section{G. BJöRCK, Distributions of positive mass}

Theorem 3. For any $\lambda>0$ and $n>1$, the support of any maximal distribution is a subset of the boundary of $F$.

Proof: As remarked by PóLYa and Szeqö [3, p. 20], simple calculation shows that for fixed $y,|x-y|^{\lambda}$ is a subharmonic function of $x$, and we see that it is even strictly subharmonic. Hence $p_{\mu}(x)=\int_{F}|x-y|^{\lambda} d \mu(y)$ is strictly subharmonic.

But then if $x$ is an inner point of $F, p_{\mu}(x)$ is strictly less than the mean value of $p_{\mu}$ on a sphere in $F$ with centre $x$. According to theorem 2 , this mean value is less than or equal to $M$. Hence, again according to theorem $2, x$ cannot belong to the support of $\mu$. This completes the proof.

Theorem 4. If $0<\lambda<2$, the maximal distribution is unique.

Proof: Suppose that $\mu$ and $\mu_{1}$ are two maximal distributions. Let $\nu=\mu_{1}-\mu$. Then $v$ satisfies the conditions (1). Hence, as in the proof of theorem 2, $v$ satisfies (2). But

$$
M=I(\mu+\nu)=I(\mu)+I(\nu)+2 \cdot I(\mu, \nu)=M+I(\nu)+2 \cdot I(\mu, \nu) .
$$

Hence

$$
I(\nu)=-2 I(\mu, \nu) \geq 0
$$

To complete the proof, it is sufficient to prove the following lemma 1. The above $\nu$, in fact, satisfies the assumptions of the lemma, and hence is identical to zero, that is $\mu_{1} \equiv \mu$.

Lemma 1. If $0<\lambda<2$ and if $v$ is a distribution on $F$ with total algebraic mass equal to zero and a non-negative energy integral, that is. if

$$
\left.\begin{array}{l}
\int_{F} d \nu=0 \\
I(\nu) \geq 0
\end{array}\right\}
$$

then $\nu$ vanishes identically.

Proof: If $n=1$, replace $n$ by 2 , that is, imbed $R_{1}$ in $R_{2}$. According to FrostMaN [2, p. 6], the formula (2) of [2] is valid for $0<\alpha+\beta<m+2, \alpha+\beta \neq m$. Apply this formula with $m=n$ (modified as indicated), $\alpha=\beta=\frac{n+\lambda}{2}$, and $\mu=\nu$. We get

$$
\int_{R_{n}}\left(U^{\alpha}\right)^{2} d z=\frac{1}{H_{n}(n+\lambda)} \cdot I(\nu) .
$$

Here $d z$ is the element of volume,

$$
U^{\alpha}=\text { const. } \times \int_{F}|x-y|^{\frac{\lambda-n}{2}} d \nu(y),
$$

and 
ARKIV FÖR MATEMATIK. Bd $3 \mathrm{nr} 21$

$$
H_{n}(n+\lambda)=\frac{\pi^{\frac{n}{2}} \cdot 2^{n+\lambda} \cdot \Gamma\left(\frac{n+\lambda}{2}\right)}{\Gamma\left(-\frac{\lambda}{2}\right)}
$$

But $\Gamma\left(-\frac{\lambda}{2}\right)<0$, and hence $H_{n}(n+\lambda)<0$. Since $I(v) \geq 0$, this implies that $U^{\alpha}=0$ almost everywhere in $R_{n}$. Hence $\nu \equiv 0$ (see M. RIEsz [4, no. 10]). This completes the proof of the lemma and of theorem 4 .

Theorem 5. If $\lambda=2$, and if $F$ is a solid sphere with radius $r$ and boundary $S$, then $M=2 r^{2}$, and a distribution is maximal if and only if its support is a subset of $S$ and its centre of gravity is the centre of $F$.

Proof: Let $\mu$ be a maximal distribution with support $F_{1}$, and let $y \in F_{1}$. Multiplying (3) by $d \mu(y)$ and integrating over $F_{1}$ we obtain

$$
I(\mu)=2 \int_{F_{1}}\left(\sum_{i=1}^{n}\left(x^{i}\right)^{2}\right) d \mu(x)-2 \sum_{i=1}^{n}\left(\int_{F_{1}} x^{i} d \mu(x)\right)^{2}
$$

We suppose now that the coordinate system is chosen with its origin in the centre of $F$. Then the first term of $I(\mu)$ is maximal $=2 r^{2}$, if and only if $F_{1} \subset S$. The modulus of the second term is minimal $=0$, if and only if

$$
\int_{F_{1}} x^{i} d \mu(x)=0, \quad(i=1,2, \ldots, n) .
$$

Hence $I(\mu)$ is maximal $=2 r^{2}$ if and only if $F_{1} \subset S$ and the origin is the centre of gravity of $\mu$, which completes the proof.

Theorem 6. If $\lambda=2$ and $F$ is contained in a solid sphere $K$ with radius $r$ and boundary $S$, and if $r$ is the minimum radius possible under these conditions, then $M=2 r^{2}$, the support of any maximal distribution is a subset of $S \cap F$, and there exists a maximal distribution the support of which consists of not more than $(n+1)$ points.

Proof: Since $F \subset K, m_{F} \subset m_{K}$. Hence $M=M_{F} \leq M_{K}=2 r^{2}$. If we can find a distribution $\mu \in M_{F}$ with $I(\mu)=2 r^{2}$, such that its support consists of not more than $(n+1)$ points, then the theorem is proved. Indeed the existence of such a distribution implies that $M=2 r^{2}$. Hence every maximal distribution for $F$ is also a maximal distribution for $K$. According to theorem 5, its support is then a subset of $S$.

Suppose that the centre $c$ of $K$ is an outer point of the convex hull of $F \cap S$. Then some half-sphere $H$ of $S$ does not meet $F$. Hence, since $F$ and $H$ are compact sets, they must have a positive distance. It is then possible, by moving $c$ towards $F$ and then shrinking the translated $K$, to construct a solid sphere containing $F$ and with radius less than $r$. This is contrary to the assumptions of the theorem. Hence $c$ belongs to the convex hull of $F \cap S$ and consequently is an inner point of a $k$-dimensional simplex with vertices 


\section{G. BJöRCK, Distributions of positive mass}

$y_{0}, y_{1}, \ldots, y_{k}$ in $F^{\prime} \cap S,(1 \leq k \leq n)$. The barycentric coordinates of $c$ in this simplex are consequently positive and are denoted by $m_{0}, m_{1}, \ldots, m_{k}$. We shall now prove that the required distribution is given by

$$
\mu=\sum_{i=0}^{k} m_{i} \delta_{y_{i}}
$$

From the properties of barycentric coordinates we know that $\sum_{i=0}^{k} m_{i}=1$, and that $c$ is the centre of gravity of $\mu$. The first of these facts, together with the inequalities $m_{t}>0$, shows that $\mu \in m_{K}$. The other, together with the fact that the support of $\mu$ is a subset of $S$, then ensures, by theorem 5 , that $I(\mu)=2 r^{2}$, which completes the proof.

Theorem 7. If $\lambda>2$ and if $F$ is a solid sphere with radius $r$, then $M=2^{\lambda-1} r^{\lambda}$, and a distribution is maximal if and only if it consists of two point-masses, each with the mass $\frac{1}{2}$, placed in the two end points of a diameter.

Proof: For any $\mu \in m$,

$$
\begin{aligned}
I(\mu) & =\iint_{F}|x-y|^{\lambda} d \mu(x) d \mu(y)=\iint_{F}|x-y|^{\lambda-2}|x-y|^{2} d \mu(x) d \mu(y) \\
& \leq(2 r)^{\lambda-2} \iint_{F}|x-y|^{2} d \mu(x) d \mu(y) \leq(2 r)^{\lambda-2} \sup _{\mu \in m} \int_{F} \int_{F}|x-y|^{2} d \mu(x) d \mu(y) \\
& =(2 r)^{\lambda-2} \cdot 2 r^{2}=2^{\lambda-1} r^{\lambda} .
\end{aligned}
$$

Hence

$$
M \leq 2^{\lambda-1} r^{\lambda}
$$

But a distribution $\mu^{*}$ consisting of the mass $\frac{1}{2}$ in each of the two end points of a diameter, gives:

and consequently,

$$
I\left(\mu^{*}\right)=2 \cdot \frac{1}{2} \cdot \frac{1}{2} \cdot(2 r)^{\lambda}=2^{\lambda-1} r^{\lambda}
$$

$$
M=2^{\lambda-1} r^{\lambda}
$$

Either the first inequality sign of (4) gives a strict inequality, or $|x-y|=2 r$ for all pairs of elements of mass that contribute to the integral $\iint_{F}|x-y|^{2} d \mu(x) d \mu(y)$, that is, for all pairs such that $|x-y| \neq 0$. Hence the only type of distribution that gives equality instead of the first inequality of (4) is the $\mu^{*}$-type. This completes the proof.

Corollary. Suppose that $\lambda>2$ and that $F$ has a diameter (that is a segment of maximum length with its end points in $F$ ) of length $2 r$ such that $F$ is contained in the solid sphere with this diameter. Then $M=2^{\lambda-1} r^{\lambda}$, and the distribution consisting of the mass $\frac{1}{2}$ in each of the end points of the diameter, is maximal. In particular, this is true for any $F$ that is centrally symmetric. If, in addition, we assume that $F$ has no other diameter, we obtain the result that the maximal distribution is unique. 
ARKIV För MATEMATIK. Bd $3 \mathrm{nr} 21$

Remark 1 (cf. Pólya and Szegö [3, p. 42]). We now know all the maximal distributions for any $\lambda>0$, when $F$ is a solid sphere (or a sphere). In fact, it follows from theorems 3 and 4 , by reasons of symmetry, that, for $0<\lambda<2$, the unique maximal distribution is the homogeneous distribution on the boundary. Further, for $\lambda>2$ the mass of a maximal distribution is concentrated in two diametrically opposite points, and for $\lambda=2$ both these types of distributions and, in addition, a great variety of intermediate forms are maximal, as specified in theorem 5. This tendency of the mass to concentrate in points when $\lambda$ increases, together with the last result of theorem 6 , suggests a hypothesis, stated and proved in theorem 12.

Remark 2. From theorems 5 and 7 it is clear that, for $\lambda \geq 2$, the maximal distribution is not necessarily unique. In fact, the solid sphere is an example of a set $F$ with an infinity of maximal distributions. However, for $\lambda>2$ all these are congruent. An example of a set $F$ with two non-congruent maximal distributions is given in remark 2 to theorem 10 .

Theorem 8. If $\lambda>1$ and if $P$ is an inner point of a $k$-dimensional simplex $(1 \leq k \leq n)$, then a positive point-mass in $P$ can be replaced by positive pointmasses in the vertices of the simplex, with the same total mass, in such a way that the potential strictly increases in every point of $R_{n}$.

Proof: Let the vertices be $x_{0}, x_{1}, \ldots, x_{k}$, and let the barycentric coordinates of $P$ in the simplex be $m_{0}, m_{1}, \ldots, m_{k}$. Since $P$ is an inner point they are all positive. We lose no generality if we assume that the original mass in $P$ is unity, that is, that the original distribution is $\delta_{P}$. We want to prove that the distribution $\mu=\sum_{i=0}^{k} m_{i} \delta_{x_{i}}$ solves the problem, that is, that in every point $y$ of $R_{n}$,

or

$$
\begin{gathered}
p_{\mu}(y)>p_{\delta_{P}}(y) \\
\sum_{i=0}^{k} m_{i}\left|y-x_{i}\right|^{\lambda}>\left|y-\sum_{i=0}^{k} m_{i} x_{i}\right|^{\lambda}
\end{gathered}
$$

But since $m_{i}>0$ and $\sum_{i=0}^{k} m_{i}=1$, this follows from the fact that for fixed $y,|x-y|^{\lambda}$ is a strictly convex function of $x$. This in turn is a consequence of the facts that $|x-y|$ is a non-negative convex function of $x$, not constant on any segment, and that $t^{\lambda}$ is a monotonously increasing, strictly convex function of the nonnegative real variable $t(\lambda>1)$. This completes the proof.

Theorem 9. If $\lambda>1$, the support of any maximal distribution consists only of extreme points of the convex hull of $F$. (An extreme point of a convex set is a point which is not an inner point of any segment with end points in the set.)

Proof: Suppose that the theorem does not hold. Then there exists a maximal distribution $\mu$ such that the interior $S$ of some $k$-dimensional simplex $(1 \leq k \leq n)$, with its vertices $x_{0}, x_{1}, \ldots, x_{k}$ in $F$, carries mass. We then "integrate 


\section{G. BJöRCK, Distributions of positive mass}

the procedure of theorem 8 over $S^{\prime \prime}$, that is, we replace the part of $\mu$ which lies in $S$ by the distribution $\sum_{i=0}^{k} m_{i} \delta_{x_{i}}$, where

$$
m_{i}=\int_{S} \xi_{i}(y) d \mu(y)
$$

with $\xi_{i}(y)=$ the barycentric coordinates of $y$ in $S(i=0,1, \ldots, k)$. If the new distribution obtained from $\mu$ in this way is denoted by $\mu_{1}$, theorem 8 assures that in any point of $R_{n}$,

Hence,

$$
p_{\mu_{1}}>p_{\mu}
$$

$$
\begin{aligned}
I\left(\mu_{1}\right)=\int p_{\mu_{1}}(x) d \mu_{1}(x)>\int & p_{\mu}(x) d \mu_{1}(x)=I\left(\mu, \mu_{1}\right) \\
= & \int p_{\mu_{1}}(x) d \mu(x)>\int p_{\mu}(x) d \mu(x)=I(\mu)=M,
\end{aligned}
$$

which is a contradiction, since $\mu_{1} \in M$. This completes the proof.

Theorem 10. Suppose that $\lambda>2$ and that $S$ is a finite set of more than $(n+1)$ points, all carrying point-masses of the distribution $\mu \in M$. Then it is possible, just by moving mass between the points of $S$, to construct a distribution $\mu^{\prime} \in M$ such that not more than $(n+1)$ points of $S$ carry masses, and such that $I\left(\mu^{\prime}\right)>I(\mu)$.

For the proof of this theorem we will need the following two lemmas:

Lemma 2. Let $y_{0}, y_{1}, \ldots, y_{m}$ be distinct points of $R_{n}$ and let $0<\alpha<2$. Then the quadratic form

$$
f=\sum_{i, k=1}^{m}\left(\left|y_{0}-y_{i}\right|^{\alpha}+\left|y_{0}-y_{k}\right|^{\alpha}-\left|y_{i}-y_{k}\right|^{\alpha}\right) t_{i} t_{k}
$$

is positive definite.

This was proved by Schomenerg [6, theorem 2]. Another proof is obtained from lemma 1 with $\lambda=\alpha$ and $\nu=\sum_{i=0}^{m} t_{i} \delta_{y_{i}}$, where $\sum_{i=0}^{m} t_{i}=0$. Using the latter relation to eliminate $t_{0}$, we get:

$$
\begin{array}{r}
I(v)=\sum_{i=0}^{m} \sum_{k=0}^{m} t_{i} t_{k}\left|y_{i}-y_{k}\right|^{\alpha}=\sum_{i=1}^{m} t_{i}\left(\sum_{k=0}^{m} t_{k}\left|y_{i}-y_{k}\right|^{\alpha}\right)-\sum_{i=1}^{m} t_{i}\left(\sum_{k=0}^{m} t_{k}\left|y_{0}-y_{k}\right|^{\alpha}\right) \\
=\sum_{i=1}^{m} t_{i}\left(\sum_{k=1}^{m} t_{k}\left|y_{i}-y_{k}\right|^{\alpha}-\sum_{k=1}^{m} t_{k}\left|y_{i}-y_{0}\right|^{\alpha}-\sum_{k=0}^{m} t_{k}\left|y_{0}-y_{k}\right|^{\alpha}\right)=-f .
\end{array}
$$

Hence $f \leq 0$ implies $I(\nu) \geq 0$. By lemma 1 , this gives $t_{i}=0 \quad(i=0,1, \ldots, m)$, which completes the proof of lemma 2.

Lemma 3. Let $a_{i k}=a_{k i}(i \neq k ; i, k=0,1, \ldots, m)$ be $\frac{1}{2} m(m+1)$ given positive numbers. Then a necessary and sufficient condition that there exist in $R_{r}$, but not in $R_{\tau-1},(m+1)$ points $x_{0}, x_{1}, \ldots, x_{m}$, such that $\left|x_{i}-x_{k}\right|=a_{i k},(i \neq k)$, is that the quadratic form 
ARKIV För MATEMATIK. Bd $3 \mathrm{nr} 21$

$$
\sum_{i, k=1}^{m}\left(a_{0 i}^{2}+a_{0 k}^{2}-a_{i k}^{2}\right) t_{i} t_{k} \quad\left(\text { with } a_{i i}=0\right)
$$

is non-negative and of rank $r$.

This result was obtained by MENGER. A simple proof is given by ScHOENBERG [5, theorem 1].

Proof of theorem 10: We prove the following statement, which is clearly equivalent to theorem 10: Suppose that the $(m+1)$ points $y_{0}, y_{1}, \ldots, y_{m}$ carry point-masses of a distribution $\mu_{1} \in M$. Suppose further that it is not possible, just by moving mass between the $y_{i}$, to construct $a$ distribution $\mu_{2} \in M$ such that at least one of the $y_{i}$ is free from mass and such that $I\left(\mu_{2}\right)>I\left(\mu_{1}\right)$. Then $m \leq n$.

Let $t_{0}, t_{1}, \ldots, t_{m}$ be a set of real numbers with sum zero: $\sum_{i=0}^{m} t_{i}=0$, and denote by $p_{i}$ the potential of $\mu_{1}$ at the point $y_{i}(i=0,1, \ldots, m): p_{i}=p_{\mu_{1}}\left(y_{i}\right)$. Define the distribution $\mu_{2}$ as follows:

We get

$$
\mu_{2}=\mu_{1}+\sum_{i=0}^{m} t_{i} \delta_{y_{i}}
$$

$$
\begin{aligned}
I\left(\mu_{2}\right)=I\left(\mu_{1}\right)+2 \sum_{i=0}^{m} t_{i} I\left(\mu_{1}, \delta_{y_{i}}\right) & +\sum_{i=0}^{m} \sum_{k=0}^{m} t_{i} t_{k} I\left(\delta_{y_{i}}, \delta_{y_{k}}\right) \\
& =I\left(\mu_{1}\right)+2 \sum_{i=0}^{m} t_{i} p_{i}+\sum_{i=0}^{m} \sum_{k=0}^{m} t_{i} t_{k}\left|y_{i}-y_{k}\right|^{2}
\end{aligned}
$$

Hence, using $t_{0}=-\sum_{i=1}^{m} t_{i}$, we get (as in the proof of lemma 2)

$$
I\left(\mu_{1}\right)-I\left(\mu_{2}\right)=L+Q
$$

where the linear form $L$ and the quadratic form $Q$ are as follows:

$$
\begin{aligned}
& L=2 \sum_{i=1}^{m} t_{i}\left(p_{0}-p_{i}\right), \\
& Q=\sum_{i, k=1}^{m}\left(\left|y_{0}-y_{i}\right|^{\lambda}+\left|y_{0}-y_{k}\right|^{\lambda}-\left|y_{i}-y_{k}\right|^{\lambda}\right) t_{i} t_{k} .
\end{aligned}
$$

We shall now prove that $Q$ is non-negative. Suppose that this is not true. Then there exists a set of $t$-values, $t_{i}=T_{i}(i=1,2, \ldots, m)$, giving $Q$ a negative value. If $k$ is a real number which is not zero, then $Q$ is negative for the set $k \cdot T_{i}$. By restricting $k$ either to positive or to negative values we can assure that $L$ is non-positive for the considered set. Hence $I\left(\mu_{2}\right)>I\left(\mu_{1}\right)$ for all positive or for all negative values of $k$. If $|k|$ is sufficiently small, the corresponding $\mu_{2} \in M$ ( $\mu_{2}$ has no negative masses). If, according to the restriction, $k$ increases or decreases from zero, it will reach a first value for which the mass of $\mu_{2}$ in one of the points $y_{i}$ is zero, and still $\mu_{2} \in M$ and $I\left(\mu_{2}\right)>I\left(\mu_{1}\right)$. This is contrary to our assumptions. Hence we have proved that $Q$ is non-negative. 


\section{G. BJöRCK, Distributions of positive mass}

We now apply lemma 3 to $a_{i k}=\left|y_{i}-y_{k}\right|^{\frac{\lambda}{2}}$.

As a result, in some $R_{r}$ there exist $(m+1)$ points $x_{0}, x_{1}, \ldots, x_{m}$, such that

$$
\left|x_{i}-x_{k}\right|=\left|y_{i}-y_{k}\right|^{\frac{\lambda}{2}}
$$

We then apply lemma 2 to the points $x_{0}, x_{1}, \ldots, x_{m}$ of $R_{r}$ with $\alpha=\frac{4}{\lambda}$.

As a result, the form

$$
\begin{aligned}
\sum_{i, k=1}^{m}\left(\left|x_{0}-x_{i}\right|^{\frac{4}{\lambda}}+\left|x_{0}-x_{k}\right|^{\frac{4}{\lambda}}-\mid\right. & \left.x_{i}-\left.x_{k}\right|^{\frac{4}{\lambda}}\right) t_{i} t_{k}= \\
& =\sum_{i, k=1}^{m}\left(\left|y_{0}-y_{i}\right|^{2}+\left|y_{0}-y_{k}\right|^{2}-\left|y_{i}-y_{k}\right|^{2}\right) t_{i} t_{k}
\end{aligned}
$$

is positive definite. Hence its rank is $m$.

We finally apply lemma 3 again, but this time to $a_{i k}=\left|y_{i}-y_{k}\right|$.

As a result, there do not exist in $R_{m-1},(m+1)$ points $z_{0}, z_{1}, \ldots, z_{m}$, such that $\left|z_{i}-z_{k}\right|=a_{i k}(i \neq k)$. However, in $R_{n}$ such points do exist, for instance $z_{i}=y_{i}$. Hence $n>m-1$, which completes the proof of theorem 10 .

Applying theorem 10 to a maximal distribution, we get the following

Corollary. For $\lambda>2$, no maximal distribution contains more than $(n+1)$ pointmasses.

Remark 1. Theorem 10 is the best possible in the sense that no smaller number can be substituted for $(n+1)$. This is shown by the following example. Let $F$ be the $(n+1)$ vertices of a regular $n$-simplex and let $\mu$ be a maximal distribution on $F$. By theorem 2, the potential takes equal values at two points carrying mass. But, due to the symmetry, the contributions from the mass in any third point to the potential at these two points are equal. Hence the masses in the two considered points must be equal. If some vertex should carry no mass, then it would be possible to increase $I(\mu)$, for example by moving a half of one of the point-masses to the empty vertex. But this contradicts the fact that $\mu$ is maximal. Hence this special $F$ has, for any $\lambda>0$, a unique maximal distribution, viz. the mass $1 /(n+1)$ in each vertex.

Remark 2. With the aid of theorem 10, we can easily show that theorem 4 fails completely for $\lambda \geq 2$, if $n>1$. For $\lambda=2$, this follows from theorem 5 , and for $\lambda>2$ we shall now give an example of a set $F$ with two maximal distributions, one consisting of two point-masses and the other of $(n+1)$ point-masses.

Let $x_{0}, x_{1}, \ldots, x_{n}$ be the vertices of a regular $n$-simplex with edges of unit length and let $c$ be its centre of gravity. Let $y$ be a point on the straight line $x_{0} c$, such that $\left|x_{0}-y\right|=\left(\frac{2 n}{n+1}\right)^{\frac{1}{\lambda}}$, and such that $c$ lies between $x_{0}$ and $y$. Let $F$ consist of the $(n+2)$ points $y, x_{0}, x_{1}, \ldots, x_{n}$. According to theorem 10 , any maximal distribution $\mu$ on $F$ will leave at least one of the points free from mass. 
Suppose first that $y$ is free from mass. Then $\mu$ is a maximal distribution on the vertices of a regular $n$-simplex, and hence, by remark 1 , each $x_{i}$ $(i=0,1, \ldots, n)$ carries the mass $1 /(n+1)$, and

$$
I(\mu)=\frac{n(n+1)}{(n+1)^{2}}=\frac{n}{n+1} .
$$

Suppose next that some $x_{i}(i \neq 0)$ is free from mass. By the same argument of symmetry as in remark 1, every $x_{i}(i \neq 0)$ is then free from mass. The support of $\mu$ thus consists of $y$ and $x_{0}$, and hence $\mu$ must have the mass $\frac{1}{2}$ in each of these two points, and $I(\mu)=2 \cdot \frac{1}{2} \cdot \frac{1}{2} \cdot\left|x_{0}-y\right|^{2}=n /(n+1)$.

Suppose finally that $y$ carries mass but $x_{0}$ does not. Then $\mu$ is not maximal. In fact, the distance from a vertex of a regular $n$-simplex with edges of unit length to the opposite $(n-1)$-dimensional face is $=\left(\frac{1+n}{2 n}\right)^{\frac{1}{2}}>\frac{1}{2} \cdot 2^{\frac{1}{2}}$, and $\left|x_{0}-y\right|$ $=\left(\frac{2 n}{n+1}\right)^{\frac{1}{\lambda}}<\left(\frac{2 n}{n+1}\right)^{\frac{1}{2}}<2^{\frac{1}{2}}$. Hence $y$ is nearer to the face opposite to $x_{0}$ than $x_{0}$ is, which means that $\left|y-x_{i}\right|<\left|x_{0}-x_{i}\right|(i=1,2, \ldots, n)$. Then $I(\mu)$ will increase if the mass of $y$ is moved to $x_{0}$.

To sum up, we know that a maximal distribution does exist, and we know that the only two distributions which may be maximal, have the same $I$-value. Hence they are both maximal.

Theorem 11. For $\lambda>2$, let $\mu$ be a maximal distribution and $K$ a compact subset of $F$. Then there exists a maximal distribution $\mu^{*}$ which in $K$ has only point-masses and which outside of $K$ is equal to $\mu$.

Proof: Since $K$ is compact, we can cover it with a finite set of solid spheres $C_{1}, \ldots, C_{p}$ with radius $\varepsilon$ and with their centres belonging to $K$. Then we construct $p$ pair-wise disjoint Borel sets $B_{1}, \ldots, B_{p}$ as follows:

$$
\begin{aligned}
& B_{1}=C_{1} \cap K \\
& B_{i}=C_{i} \cap K-C_{i} \cap\left(\bigcup_{j<i} B_{j}\right) \quad(1<i \leq p) .
\end{aligned}
$$

Denote by $\mu_{0}$ the part of $\mu$ in $F-K$, by $m_{i}$ the mass of $\mu$ in $B_{i}$, and by $\delta_{i}$ the distribution consisting of the unit mass in the centre of $C_{i}$. We then define a distribution $\mu_{\varepsilon} \in M$ as follows:

$$
\mu_{\varepsilon}=\mu_{0}+\sum_{i=1}^{p} m_{i} \delta_{i}
$$

that is, we concentrate into $p$ points of $K$ the mass of $\mu$ in $K$. Since no mass is moved a longer distance than $\varepsilon,|x-y|^{\lambda}$ does not change more than by the amount $k \cdot \varepsilon$, where $k$ is a constant, depending only on $F$ and $\lambda$, for since $t^{\lambda}$ is an increasing convex function for $t>0$, the change of $|x-y|^{\lambda}$ is less than $(d+\varepsilon)^{\lambda}-(d-\varepsilon)^{\lambda}<\lambda(2 d)^{\lambda-1} \cdot 2 \varepsilon$, where $d$ is the diameter of $F$. Hence the value of $I$ does not change more than by the amount $k \cdot \varepsilon$, that is,

$$
I\left(\mu_{\varepsilon}\right) \geq M-k \cdot \varepsilon .
$$




\section{G. BJÖRCK, Distributions of positive mass}

We now take as the set $S$ and the distribution $\mu$ of theorem 10 , the $p$ centres of the solid spheres $C_{i}$ and the distribution $\mu_{\varepsilon}$, respectively. By theorem 10, there is then a distribution $\mu_{\varepsilon}^{\prime} \in M$ of the form

$$
\mu_{\varepsilon}^{\prime}=\mu_{0}+\sum_{i=1}^{p} m_{i}^{\prime} \delta_{i}
$$

with not more than $(n+1)$ of the non-negative numbers $m_{i}^{\prime}$ different from zero, and such that

By (5), this implies

$$
I\left(\mu_{\varepsilon}^{\prime}\right) \geq I\left(\mu_{\varepsilon}\right)
$$

$$
M \geq I\left(\mu_{\varepsilon}^{\prime}\right) \geq M-k \cdot \varepsilon .
$$

Those $m_{i}^{\prime}$ which are positive, together with a sufficient number of zeroes, are now renamed $m_{0}^{(\varepsilon)}, m_{1}^{(\varepsilon)}, \ldots, m_{n}^{(\varepsilon)}$. The corresponding points, and, in the case of the zeroes, some arbitrary points of $K$, are denoted by $x_{0}^{(\varepsilon)}, x_{1}^{(\varepsilon)}, \ldots, x_{n}^{(\varepsilon)}$.

We now choose a sequence of $\varepsilon$, tending towards zero. For each $\varepsilon$ we construct the points $x_{0}^{(\varepsilon)}, x_{1}^{(\varepsilon)}, \ldots, x_{n}^{(\varepsilon)}$ of $K$ and the non-negative numbers $m_{0}^{(\varepsilon)}, m_{1}^{(\varepsilon)}$, $\ldots, m_{n}^{(\varepsilon)}$ with sum $\mu(K)$. Since both $K$ and the closed interval $[0, \mu(K)]$ are compact, it is possible to select a sub-sequence $\varepsilon_{\nu}$ such that the point sequences $\left\{x_{i}^{\left(\varepsilon_{\nu}\right)}\right\}_{v}$ and the number sequences $\left\{m_{i}^{\left(\varepsilon_{\nu}\right)}\right\}_{p}$ all converge. The limit elements are denoted by $x_{i}^{*}$ and $m_{i}^{*}$ and belong to $K$ and $[0, \mu(K)]$, respectively. We also have $\sum_{i=0}^{n} m_{i}^{*}=\mu(K)$. Of course some of the $m_{i}^{*}$ may be zero. We define the distribution $\mu^{*}$ as follows:

$$
\mu^{*}=\mu_{0}+\sum_{i=0}^{n} m_{i}^{*} \delta_{x_{i}}^{*}
$$

We have just proved that

$$
\mu^{*} \in M
$$

But by choosing $\nu$ sufficiently large, we can make $\left|m_{i}^{*}-m_{i}^{\left(\epsilon_{v}\right)}\right|$ and $\left|x_{i}^{*}-x_{i}^{\left(\epsilon_{\nu}\right)}\right|$, $(i=0,1, \ldots, n)$, arbitrarily small. Hence, since $I\left(\mu_{\varepsilon}^{\prime}\right)$ is a continuous function of the variables $x_{i}^{(\varepsilon)}$ and $m_{i}^{(\varepsilon)},\left|I\left(\mu^{*}\right)-I\left(\mu_{\varepsilon_{v}}^{\prime}\right)\right|$ can be made arbitrarily small. By $(6)$, this implies

$$
I\left(\mu^{*}\right)=M
$$

This completes the proof that $\mu^{*}$ satisfies the requirements of the theorem.

Theorem 12. If $\lambda>2$, every maximal distribution consists entirely of pointmasses, not more than $(n+1)$ in number, and all situated in extreme points of the convex hull of $F$.

Proof: The last part of the theorem is contained in theorem 9. All we have to prove is that the support of a maximal distribution cannot consist of more than $(n+1)$ points. Suppose that this is not true and that $x_{1}, x_{2}, \ldots, x_{n+2}$ are distinet points of the support of a maximal distribution $\mu$. Let $C_{i}(i=1,2, \ldots, n+2)$ 
ARKIV För MATEMATIK. Bd 3 nr 21

be solid spheres with centres $x_{i}$ and so small that they are pair-wise disjoint. Define the compact sets $K_{i}$ as

$$
K_{i}=C_{i} \cap F
$$

Since $x_{i}$ is in the support of $\mu, \mu\left(K_{i}\right) \neq 0$.

We now apply theorem 11 with $K=K_{1}$. We obtain a maximal distribution $\mu_{1}^{*}$ which is equal to $\mu$ except in $K_{1}$ and which has at least one point-mass in $K_{1}$.

We then apply theorem 11 to $\mu_{1}^{*}$ instead of to $\mu$ and with $K=K_{2}$. We obtain a maximal distribution $\mu_{2}^{*}$, which, except in $K_{2}$, is equal to $\mu_{1}^{*}$, and which has at least one point-mass in $K_{1}$ and one in $K_{2}$.

We go on in this way and finally obtain a maximal distribution $\mu_{n+2}^{*}$, which contains at least $(n+2)$ point-masses. This contradicts the corollary of theorem 10, which completes the proof.

With theorem 12 we conclude the treatment of our main subject. We now turn our interest to the relations between the maximal distributions and the distributions solving the two other extremum problems mentioned in the introduction, namely the distributions $\mu \in m$ for which

or

$$
\begin{aligned}
& \max _{x \in F} p_{\mu}(x)=\min _{\mu \in m} \max _{x \in F} p_{\mu}(x)=M^{\prime} \\
& \min _{x \in F} p_{\mu}(x)=\max _{\mu \in m} \min _{x \in F} p_{\mu}(x)=M^{\prime \prime} .
\end{aligned}
$$

Let us call such distributions minmax and maxmin distributions, respectively. There evidently always exists at least one maxmin distribution and one minmax distribution, and $M^{\prime \prime} \leq M$.

From theorem 8 it follows that, for $\lambda>1$, a maxmin distribution must have all its mass in the extreme points of the convex hull of $F$. Otherwise the potential at every point of $R_{n}$, and hence also its minimum value on $F$, could be increased by moving the masses.

We might thus suspect that every maximal distribution is also maxmin, or conversely. However, this is not true for any $\lambda>0$, as is shown by the following theorems and examples, which also rule out the corresponding hypothesis for the minmax distributions.

Theorem 13. When $F$ is a sphere, the potential of any minmax or maxmin distribution is constant on $F$.

Proof for the maxmin case:

Consider for every maxmin distribution $\mu$ the set $F_{\mu}$ of all points $x \in F$ such that $p_{\mu}(x)=M^{\prime \prime}$. These $F_{\mu}$ are closed. The intersection of any finite number of sets $F_{\mu}$ is non-empty. In fact, if there is no point where $p_{\mu_{1}}, \ldots, p_{\mu_{n}}$ are all equal to their minimum value $M^{\prime \prime}$, then the distribution $\frac{1}{n} \sum_{i=1}^{n} \mu_{i}$ has a minimum potential greater than $M^{\prime \prime}$, which is impossible. Hence, since $F$ is compact, there is a point belonging to every $F_{\mu}$. Then, by reasons of symmetry, every 


\section{G. BJöRCK, Distributions of positive mass}

point of $F$ belongs to every $F_{\mu}$, which completes the proof. Hence, when $\lambda>2$ and $F$ is a sphere, the maximal and the maxmin distributions are different.

To deal with the maxmin distributions for $0<\lambda \leq 2$, we consider a set $F$ consisting of a sphere $S$ and a point $y$ inside $S$, but not its centre. A maximal distribution $\mu$ must have its minimum potential on $F$ at $y$ (since $p_{\mu}$ is subharmonic), and this minimum is strictly less than the constant value of the potential on $S$. Hence by moving the masses on $S$ in a direction away from $y$, we can increase the minimum potential on $F$, and so $\mu$ is not maxmin.

To deal with the minmax distributions for any $\lambda>0$, we consider as $F$ a solid sphere with centre $c$. A maximal distribution $\mu$ has all its mass on the boundary, and hence $p_{\mu}(c)=r^{\lambda}$. But this value is strictly less than the mean value of the potential on the boundary. Hence, $\max p_{\mu}(x)>r^{\lambda}$. On the other hand, for the distribution $\delta_{c}$ we have $\max _{x \in F} p_{\delta_{c}}(x)=r^{\lambda}$. Hence the minmax and the maximal distributions are different.

Finally we give a certain property of minmax distributions and of a related class of distributions:

Theorem 14. When $\lambda>\mathrm{I}$ and $F$ is convex, any minmax distribution consists of one point-mass. This follows from theorem 8. In fact, the potential in every point of $R_{n}$ will strictly decrease, if the distribution is replaced by the unit mass in its centre of gravity.

Let us denote as "free minmax distribution" a distribution such that

$$
\max _{x \in F} p_{\mu}(x)=\min _{\substack{\mu \geq 0 \\ \int_{R_{n}} \delta \mu=1}} \max _{x \in F} p_{\mu}(x),
$$

that is, a distribution which solves the minmax problem where $\mu$ is not restricted to lie on $F$. Such distributions are the "Endverteilungen für $R^{(\lambda)}$ ", considered by Pólya and Szegö [3, p. 42]. There, the following result is stated in the special case when $F$ is a sphere or a closed sphere.

Theorem 15. If $\lambda>1$, any free minmax distribution consists of one point-mass.

Proof: By the preceding result, it is sufficient to prove that any free minmax distribution $\mu$ is a minmax distribution. for the convex hull $H(F)$ of $F$. As remarked by PólyA and SzEgö [3, footnote 14], the support of $\mu$ is a subset of $H(F)$. All we have to prove then, is that there is a point $z \in F^{\prime}$ such that

$$
\max _{y \in H(F)} p_{\mu}(y)=p_{\mu}(z)
$$

But this is true for any distribution $\mu \geq 0$, when $\lambda>1$. In fact, since $|x-y|^{\lambda}$ is a convex function of $x$ (theorem 8 ), so is $p_{\mu}(x)=\int_{R_{n}}|x-y|^{\lambda} d \mu(y)$, and hence there is an extreme point $z$ of $H(F)$, such that (7) is true. This completes the proof. 
ARKIV För Matematik. Bd 3 nr 21

\section{R E F E R E N G E S}

1. Frostman, O., Potentiel d'équilibre et capacité des ensembles. Medd. Lunds Univ. Mat. Sem. 3 (1935).

2. - Potentiel de masses à somme algébrique nulle. Kungl. Fysiogr. Sällsk. i Lund förhandl. 20,3-23 (1950).

3. Pólya, G. and Szeqö, G., Über den transfiniten Durchmesser (Kapazitätskonstante) von ebenen und räumlichen Punktmengen. Journal für die reine und angewandte Mathematik 165, 4-49 (1931).

4. Rigsz, M., Intégrales et potentiels. Acta Szeged 9, 1-42 (1938-40).

5. Schoenbero, I. J., Remarks to Maurice Fréchet's article "Sur la définition axionatique d'une classe d'espaces vectoriels distanciés applicables vectoriellement sur l'espace de Hilbert". Annals of Math. 36, 724-732 (1935).

6. - On certain metric spaces arising from Euclidean spaces by a change of metric and their imbedding in Hilbert space. Annals of Math. 38, 787-793 (1937). 